\title{
Comportement de cinq variétés de riz pluvial (Oryza sativa L.) à la Pyriculariose et la Verse dans les conditions naturelles à Kisangani, République Démocratique du Congo
}

\author{
Liboga Oenabaiso Bienvenu1,2, Litucha Bakokola Joseph ., Ngama Boloy Faustin²., Balimo Isecha \\ Fidele $^{3}$ Kayawa Lisamba Joseph ${ }^{1}$ \\ 1. Institut National pour l'Etude et la Recherche Agronomiques (INERA), P.B.2.015 Kisangani, R.D. Congo \\ 2. Institut Facultaire des Sciences Agronomiques de Yangambi, (IFA), B.P.28 Yangambi, R.D. Congo. \\ 3. Inspection provinciale de l'agriculture, pèche et élevage (IPAPEL), B.P.1714 Kisangani, R.D.Congo. \\ *Auteur correspondant, e-mail:bienvenuliboga@yahoo.fr ; téléphone: (+243)971888175
}

Original submitted in on $3^{\text {rd }}$ June 2019. Published online at www.m.elewa.org/journals/ on 31st January 2020 https://doi.org/10.35759/JABs.v145.11

\begin{abstract}
RESUME
Objectif: La Pyriculariose du riz, causée par Pyricularia grisea Cav. entraine de pertes importantes de rendement chez le riz. L'évaluation de comportement à la pyriculariose et à la verse de cinq variétés de riz pluvial dans les conditions naturelles à Kisangani en vue de sélectionner les meilleures était notre objectif.

Méthode et résultats: Deux essais étaient réalisés suivant le dispositif de blocs complets randomisés comportant 4 répétitions et cinq traitements (variétés) (Lioto, Liboga, Lienge, Nerica7 et Kitombe). Les observations ont porté sur l'aire de progression de maladie (AUDPC), le pourcentage de surface foliaire malade (\%SFM) et de panicules malades (\%PnM) suivant l'échelle de l'IRRI (IRRI, 2002). La verse était évaluée suivant l'échelle de I'ADRAO (ADRAO, 2009). En saison A, les moyennes d'incidence de Pyriculariose paniculaire trouvées étaient de: $3,5 \% ; 3,7 \% ; 4,47 ; 6,1 \% ; 6,5 \%$ respectivement pour la variété Liboga, Nerica7, Lioto, Lienge et Kitombe. En saison B les moyennes trouvées sont : Liboga : 3,7\%, Nerica7:3,7\%, Lioto 3,9, Kitombe 6,5\%, Lienge : 7,7\%

Conclusion et applications de résultats : La pyriculariose paniclulaire affecte directement la production du riz, par le non remplissage de grains, mauvaise sortie de panicule, réduction de poids de grains, etc. Les valeurs moyennes d'incidence de pyriculariose paniculaire obtenues par la variété Liboga et Nerica7 durant les deux essais ne dépassent pas $4 \%$, en conséquence ces variétés sont considérées comme ayant un bon niveau de résistance quantitative qui s'exprime comme une résistance de type horizontal, caractérisée par la résistance stable dans différentes conditions. La score minimale de 1, obtenue pour la verse permet de classer la variété Lioto, Liboga et Nerica7 comme résistantes et Kitombe avec la score de 5 comme moyennement sensible à la verse.
\end{abstract}

Mots clés: Riz pluvial, pyriculariose, verse, égrenage, Kisangani. 
Behavior of five varieties of upland rice (Oryza sativa L.) to blast and to lodging under natural conditions in Kisangani, République Démocratique du Congo

\section{ABSTRACT}

Objectives: rice blast caused by Pyricularia grisea causes significant yield losses in rice. Evaluation of behavior to rice blast and to lodging of five upland rice in natural conditions in order to select the best was our objective.

Methodology and Results: Two trials were carried out using randomized complete block device with 4 repetitions and five treatments or varieties. We observed the percentage of foliar diseased surface and percentage of panicle diseased as well as the area under the disease progress curve (AUDPC). Disease incidence was observed following IRRI's scale (IRRI, 2002). The logging was evaluated according to ADRAO's scale (ADRAO, 2009). In the season A mean incidence of panicle blast was: Liboga 3.5\%, Nerica7 3.7\%, Lienge $6.1 \%$ and Kitombe $6.5 \%$. In season B l'incidence of panicle blast was: Liboga 3.7\%, Nerica7 3.7\%, Kitombe 6.5\%, Lienge 7.7\%

Conclusions and application of findings: Panicle blast affects directly production of rice through non-grain filling, poor exit of panicle, and by grain weight reduction. The mean values of panicle blast incidence obtained by Liboga and Nerica7 varieties during two trials do not exceed $4 \%$, therefore these varieties are considered to have a good level of quantitative resistance that is expressed as a horizontal type resistance, characterized by stable resistance under different conditions. The minimum score of 1 obtained for lodging makes it possible to classify the variety Lioto, Liboga and Nerica7 as resistant and Kitombe with the score of 5 as moderately sensitive to lodging.

Keywords: upland rice, blast, lodging, ginning, Kisangani

\section{INTRODUCTION}

Parmi les céréales cultivées, le riz (Oryza sativa L.) est d'une grande importance en terme de consommation et de superficie cultivée (Ouédraogo et al., 2017).En RDC, la production du riz est essentiellement basée sur le riz pluvial bien que ce pays offre des potentialités énormes pour une riziculture irriguée (Bangata et al., 2013). Malgré les efforts fournis par les pouvoirs publics et des partenaires pour l'augmentation de production par la mise au point des itinéraires techniques et des variétés améliorées, la production nationale de cette denrée reste faible. Des nombreuses causes sont à la base de cette faible production, il s'agit entre autre de la qualité des semences utilisées, les perturbations climatiques, les contraintes abiotiques (Momolu et al.,2016; Gnago et al.,2017; Samota et al.,2017; Islam et al.,2018) et les contraintes biotiques. Dans ces dernières, il y a les insectes ravageurs, les mauvaises herbes (Babiker et al., 2016), les rongeurs, les oiseaux, mais surtout les maladies (Bouet et al., 2010) et où la principale en zone tropicale pour le riz pluvial reste la pyriculariose, causée par Pyricularia grisea
(Cooke) Sacc. [Magnaporthe grisea (Hebert) Barr], (Kim et al., 2003; Leila et al., 2004 ; Ashkani et al., 2016).Cette maladie cause de pertes économiques de rendement chaque année qui peuvent atteindre $30 \%$. Ces pertes peuvent dépasser les $50 \%$ en fonction de l'environnement, de la conduite de champ et de la dynamique de population de pathogène (Ashkani et al., 2016). P. grisea est un champignon polycycle qui affecte toutes les parties aériennes du riz à tous les stades et réalise un cycle complet en 6-9 jours avec 9-15 cycles par cycle végétatif. La source de l'inoculum primaire est souvent les semences ou les matériels contaminés et les résidus de champs infectés. Une autre cause de baisse de rendement chez le riz reste l'effet de verse de plants de riz aux champs. $\mathrm{Ce}$ facteur entraine également des pertes importantes de rendement surtout si elle survient à la floraison avant le remplissage de grains, en entrainant un taux élevé d'infertilité des épillets. Si elle survient à la maturité avant récolte, elle entraine la chute de grains par l'égrenage spontané, par la difficulté de récolter des plants couchés et l'incidence élevée des rongeurs 
granivores. Cependant, cet aspect n'attire pas souvent l'attention des chercheurs et même des paysans, c'est ainsi que très peu de travaux existent sur cela (Bodelmann, 1976).Dans la lutte contre la pyriculariose, on préconise la destruction de fanes des champs infectés, la désinfection du sol contaminé. Ces méthodes demeurent cependant des solutions non durables (Yu et al., 1991 ; Naqvi et al., 1995). L'utilisation des produits chimiques a révélé des résultats satisfaisants, mais, dans le contexte écologique de protection de l'environnement, les produits chimiques sont de moins en moins utilisés puisqu'ils provoquent la pollution du sol, des eaux souterraines et de l'air, ils induisent de la résistance chez l'agent combattu

\section{MATERIELS ET METHODES}

Matériels biologiques : Cinq variétés du riz pluvial dont quatre sont des variétés améliorées (Lienge, Lioto, Liboga, et Nercica7) et une variété locale Kitombe ont été utilisées. Les semences ont été commandées du centre de recherche de Yangambi (INERA-Yangambi).

Dispositif expérimental : Les essais ont été conduits suivant le dispositif en blocs complets randomisés avec 4 répétitions. Chaque répétition (bloc) comportait 5 parcelles élémentaires ( $P E)$ correspondant ainsi aux variétés étudiées, soit un total de 20 parcelles. Les dimensions de P.E étaient de $4 \mathrm{~m} \times 3 \mathrm{~m}$, soit $12 \mathrm{~m}^{2}$ ces parcelles étaient séparées les unes des autres par les allées de $0,5 \mathrm{~m}$ tandis que les blocs étaient séparés entre eux de $1 \mathrm{~m}$ telle que recommandé (Kasongo et al., 2003).Le semis direct était manuellement réalisé aux écartements de $20 \mathrm{~cm} \times 25 \mathrm{~cm}$ avec 4 grains par poquet.

Période et localisation de l'essai : Cette étude a été réalisée au site expérimental de l'Institut Facultaire des Sciences agronomiques de Yangambi à Kisangani (IFA-Yangambi) $\left(25^{\circ} 11^{\prime}\right.$ Est et $0^{\circ} 31^{\prime} \mathrm{N}$ ord, $\left.410 \mathrm{~m}\right)$. La première répétition de Novembre 2016 à Avril 2017, ce qui correspond à la saison culturale B/2016 (SB) et la deuxième répétition de Mars à Aout 2017, ce qui correspond à la saison culturale A/2017(SA) dans cette zone d'étude. L'IFA-Yangambi est situé à environ $3 \mathrm{Km}$ du centre-ville de Kisangani, R. D. Congo. Le climat de Kisangani est de type Af de la classification de Köppen. II est chaud et humide avec une moyenne de température qui oscille entre $24-25^{\circ} \mathrm{C}$ et une humidité relative de 80 à $90 \%$. Les pluies sont réparties durant presque toute l'année mais le pic se situe en Octobre et peuvent atteindre annuellement une hauteur et exposent les paysans à des intoxications (Lepoivre, 2003). La priorité reste par conséquent accordée aux bonnes pratiques agricoles (BPA) peu polluantes et respectueuses de l'environnement en vue de respecter la limite maximale de résidus (LMR) dans les produits à livrer pour la sécurité des consommateurs. La méthode la plus efficace et la moins couteuse reste l'utilisation des variétés résistantes ou tolérantes vu la situation socio-économiques de paysans (Gnago et al., 2017).Dans ce contexte, ce travail se fixe l'objectif d'évaluer le comportement de 5 variétés de riz pluvial à la Pyriculariose et la verse dans les conditions naturelles à Kisangani en vue de sélectionner les meilleures.

de $1800 \mathrm{~mm}$ et 1925 heures d'insolation. Les sols du site expérimental sont des sols ferralitiques de couleur brun noire, profonds et à prédominance d'argile, type kaolinite. Ils appartiennent à l'ordre des oxisols selon soil taxonomy (Van Wembeke et Liebens, 1957).

Observation et analyse des données : La verse a été évaluée au stade 8 à 9 (grains pâteux à maturation) avec l'échelle de cotation de l'ADRAO (ADRAO, 2009) (tableau 1).L'incidence de pyriculariose foliaire a été évaluée avec l'échelle visuelle de l'IRRI (IRRI, 2002) tous les sept jours, du 21 jusqu'à 56ème jour après semis (JAS). Les notes ont été attribuées aux 15 poquets (touffe du riz) pris sur deux diagonales de chaque P.E et ont été exprimées en pourcentage de surface foliaire malade (\%SFM).Pour les panicules, l'incidence a été exprimée en pourcentage des panicules malades (\%PnM) évaluée tous les 7 jours, du

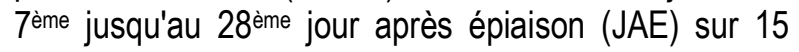
poquets pris sur deux diagonales au niveau de chaque P.E. Les échantillons des feuilles et de panicules malades prélevées au niveau de front d'avancement de la maladie ont été préparés puis observés au microscope après avoir ajouté une goutte de bleu de Lactophenol pour visualisation et la confirmation de $P$. grisea. Le taux de progression de la maladie ( $r$ ) exprimé en unité par jour (Oùedraogo, 2008) a été calculé par la relation:

$r$ (unité/jour)= $\left[\log \left(1 / 1-x_{2}\right)-\log \left(1 / 1-x_{1}\right)\right] /\left(t_{2}-t_{1}\right)$, où: $x_{1}$ et $x_{2}$ désignent les quantités de maladie exprimées en pourcentage et $\left(t_{2}-t_{1}\right)$ est l'intervalle de temps (en jours) entre deux observations. L'aire de progression de la maladie ou Area Under the disease progress curve 
(AUDPC) représente le cumul de l'incidence de la maladie sur les feuilles et de panicule durant la durée de l'observation de l'essai. II permet d'apprécier la réaction du génotype à l'ensemble des souches locales du milieu où les expérimentations ont été réalisées (Oùedraogo,2008).II a été calculée par la formule de Shaner et Finney (1977): AUDPC $=\sum\left(D i+D i_{+1} / 2\left(t_{i+1}-t_{1}\right)\right.$ où $D_{i}$ et $\mathrm{Di}_{+1}$, représentent les indices de la maladie exprimées en pourcentage de surface foliaire malade (\%SFM) observées aux temps $t_{i+1}$ et $t_{i}$, le $\left(t_{i+1}-t_{i}\right)$ représente l'intervalle de temps (en jours) entre deux observations. L'évaluation de la résistance à l'égrenage a été réalisée au stade 9 (maturation), elle a consisté à tirer doucement par la main une panicule (avec un échantillon de 10 panicules dans chaque P.E) de sa base (à partir du coup paniculaire) jusqu'au sommet et compter alors le nombre des grains qui se détachent de la panicule et qui restent dans la main, cela à la maturité $80 \%$ et $100 \%$. L'analyse de la variance (ANOVA) à un facteur a été utilisée pour la comparaison des moyennes. Lorsque les différences sont observées, elle est complétée par le Test de plus petite différence significative (LSD) pour la séparation des moyennes. Mais d'après Louvel et al. (1981) on admet qu'une variété a une résistance variétale stable lorsque la note maximale est de $4 \%$ et instable entre 6 $9 \%$ dans plusieurs localités (Notteghen, 1981) et en différentes conditions.

Tableau 1. Echelle visuelle de cotation de la verse (ADRAO, 2009)

\begin{tabular}{ccc}
\hline$\%$ des plants versés & score & Appréciation \\
\hline 0 & 0 & Résistant \\
1 & 1 & Résistant \\
1 à 5 & 3 & Moyennement résistant \\
6 à 25 & 5 & Moyennement sensible \\
26 à 50 & 7 & Sensible \\
100 & 9 & Très Sensible \\
\hline
\end{tabular}

\section{RESULTATS ET DISCUSSION}

Incidence de Pyriculariose foliaire : Dans les deux saisons, les conditions de développement de pyriculariose ont été favorables, ce qui a permis d'évaluer l'incidence de la maladie marquée par \%SFM et la de progression de la maladie dans la plante marquée par ( $r$ ).ll est à constater que les variétés de riz se comportent de façon différente en différents stades de développement de la culture (tableau 2).Au début de tallage (21JAS) certaines variétés affichent déjà les symptômes de la maladie, (Lioto, Liboga et Nerica7) excepté la variété Lienge. La croissance de la maladie s'accélère en fin tallage (42JAS) et atteint des grandes valeurs au début de montaison (56JAS) comme illustre la figure 1, probablement par ce qu'à cette phase de montaison la plante dévient fragile en consacrant beaucoup d'énergie pour la formation de grains. Ces résultats montrent par ailleurs que l'incidence de pyriculariose foliaire est élevée de façon générale en saison B qu'à la saison A suite aux conditions pluviométriques ayant prévalues en saison $B$, car il est reconnu que les conditions d'humidité permanente (Parlevliet, 1983) et de forte densité de semis (Kasongo et al., 2003) favorisent le développement de $P$. grisea.

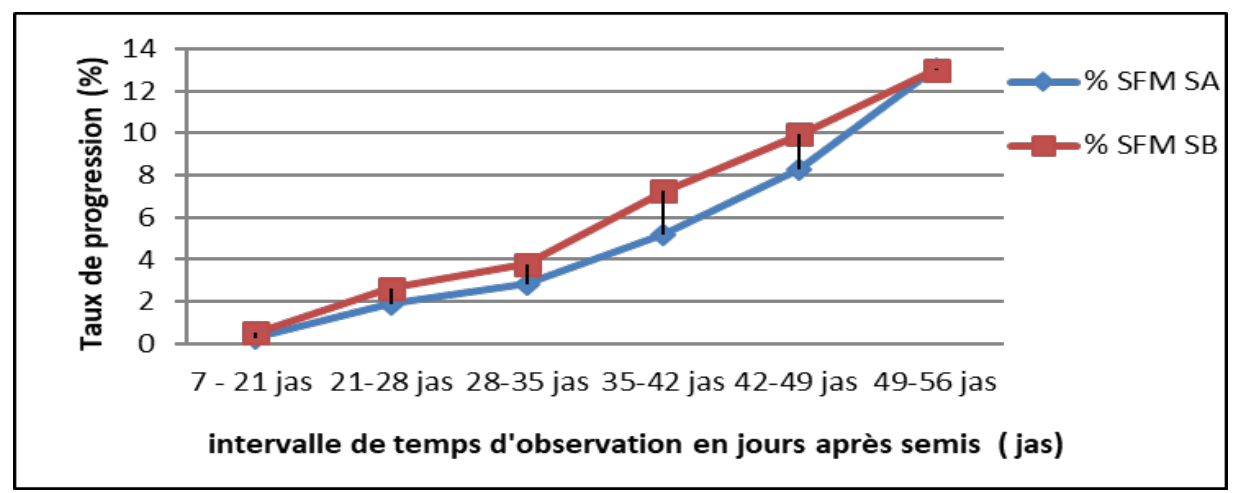

Figure 1: Courbes d'évolution de maladie sur les feuilles durant les deux saisons culturales 
Tableau 2. Incidence de pyriculariose foliaire

\begin{tabular}{|c|c|c|c|c|c|c|c|c|c|c|c|c|}
\hline \multirow[b]{2}{*}{ variété } & \multicolumn{6}{|c|}{$\%$ SFM en SA } & \multicolumn{6}{|c|}{$\%$ de progression de maladie $(r)$} \\
\hline & $\begin{array}{c}21 \\
\text { JAS }\end{array}$ & $\begin{array}{c}28 \\
\text { JAS }\end{array}$ & $\begin{array}{c}35 \\
\text { JAS }\end{array}$ & $\begin{array}{c}42 \\
\text { JAS }\end{array}$ & $\begin{array}{c}49 \\
\text { JAS }\end{array}$ & $\begin{array}{c}56 \\
\text { JAS }\end{array}$ & MV & $\mathrm{r} 1$ & r2 & r3 & r4 & r5 \\
\hline Lienge & $0,01 \mathrm{~b}$ & $0,86 \mathrm{c}$ & $3,04 b$ & $5,49 b$ & $5,31 d$ & $7,56 \mathrm{~b}$ & 3,61 & 0,04 & 0,39 & 0,35 & $-0,03$ & 0,32 \\
\hline Lioto & $0,05 b$ & $1,41 b$ & $3,15 b$ & $6,17 a$ & $7,71 \mathrm{c}$ & $12,34 a$ & 5,19 & 0,14 & 0,25 & 0,43 & 0,22 & 0,66 \\
\hline Nerica7 & $0,03 b$ & $2,48 a$ & $4,72 a$ & $7,20 \mathrm{a}$ & $10,36 \mathrm{~b}$ & $15,12 a$ & 6,6 & 0,32 & 0,32 & 0,35 & 0,45 & 0,68 \\
\hline Liboga & $0,06 b$ & $1,69 b$ & $3,08 b$ & $2,23 c$ & $12,56 a$ & $17,03 a$ & 5,7 & 0,23 & $-0,14$ & 0,22 & 1,48 & 0,64 \\
\hline Kitombe & $0,48 a$ & $2,00 a$ & $2,83 b$ & $5,13 b$ & $5,56 \mathrm{~d}$ & $13,26 a$ & 4,87 & 0,22 & 0,12 & 0,33 & 0,06 & 1,10 \\
\hline $\mathrm{CV}(\%)$ & 66,6 & 17,26 & 10,11 & 8,90 & 10,36 & 21,30 & & & & & & \\
\hline $\mathrm{S}^{2}$ & 0,20 & 1,5 & 2,37 & 2,95 & 39,18 & 50,78 & & & & & & \\
\hline \multirow[t]{2}{*}{ LSD $5 \%$} & $0,17^{* * *}$ & $0,63^{* * *}$ & $0,74^{* * *}$ & $1,14^{* * *}$ & $1,87^{* * *}$ & $6,03^{* * *}$ & & & & & & \\
\hline & \multicolumn{6}{|c|}{$\%$ SFM en SB } & \multicolumn{6}{|c|}{$\%$ de progression de maladie $(r)$} \\
\hline Lienge & $0,37 b$ & $3,64 a$ & $2,43 e$ & $5,60 \mathrm{~b}$ & $7,62 d$ & $10,70 c$ & 4,9 & 0,47 & $-0,17$ & 0,45 & 0,29 & 0,44 \\
\hline Lioto & $0,85 a$ & $2,80 b$ & $4,34 b$ & $5,38 b$ & $8,42 \mathrm{c}$ & $13,00 b$ & 5,7 & 0,28 & 0,22 & 0,15 & 0,43 & 0,66 \\
\hline Nerica7 & $0,39 b$ & $2,00 d$ & $3,02 d$ & $5,25 b$ & $6,37 d$ & $13,2 b$ & 5,0 & 0,23 & 0,15 & 0,32 & 0,16 & 0,98 \\
\hline Liboga & $0,53 b$ & $2,50 c$ & $3,73 c$ & $7,32 b$ & $11,53 b$ & $12,6 b$ & 6,3 & 0,28 & 0,18 & 0,51 & 0,60 & 0,16 \\
\hline Kitombe & $0,4 b$ & $2,27 \mathrm{c}$ & $5,39 a$ & $12,60 \mathrm{a}$ & $15,76 a$ & $15,5 a$ & 8,6 & 0,26 & 0,45 & 1,03 & 0,45 & $-0,04$ \\
\hline CV(\%) & 25,49 & 7,5 & 6,46 & 30,99 & 6,14 & 5,56 & & & & & & \\
\hline $\mathrm{S}^{2}$ & 0,15 & 0,36 & 3,19 & 155,45 & 56,79 & 11,96 & & & & & & \\
\hline LSD 5\% & $0,28^{* * *}$ & $0,39^{\star * *}$ & $0,56^{\star * *}$ & $4,90^{\star \star *}$ & $1,32^{\star \star *}$ & $1,56^{\star * *}$ & & & & & & \\
\hline
\end{tabular}

Sur la même colonne les valeurs portant les mêmes lettres ne sont pas significativement différentes au seuil de $5 \%$, $M V=$ moyenne variétale, $J A S=$ jour après semis, ${ }^{* *}=$ différence significative au seuil de $0,1 \%, S A=$ saison $A, S B=$ saison $B, S F M=$ surface foliaire malade.

Incidence de pyriculariose paniculaire : L'incidence de pyriculariose paniculaire marquée par \%PnM s'est diversement manifestées en fonction des variétés et de saison culturale (tableau 3).Toutes les variétés sont attaquées au début de remplissage de grains (7JAE) mais connaissent un accroissement élevé au stade 9 (maturation) au 28JAE telle qu'illustre la figure 2. Ceci, probablement à cause de l'état fragile de la plante dû à l'intense activité consacrée pour le murissement des grains. En saison A les valeurs moyennes les plus élevées d'incidence de pyriculariose sont observées chez les variétés Lienge $(6,1 \%)$ et Kitombe $(6,5 \%)$ alors que les plus petites valeurs sont observées chez les variétés Liboga $(3,5 \%)$ et Nerica7 $(3,7 \%)$. Les mêmes tendances sont observées en saison $B$ chez la variété Lienge $(7,7 \%)$ et Kitombe $(6,5 \%)$ contre Liboga $(3,7 \%)$ et Nerica7 (3,7\%). Ce comportement différentiel serait dû au potentiel génétique de chaque variété à résister contre les agents pathogènes.

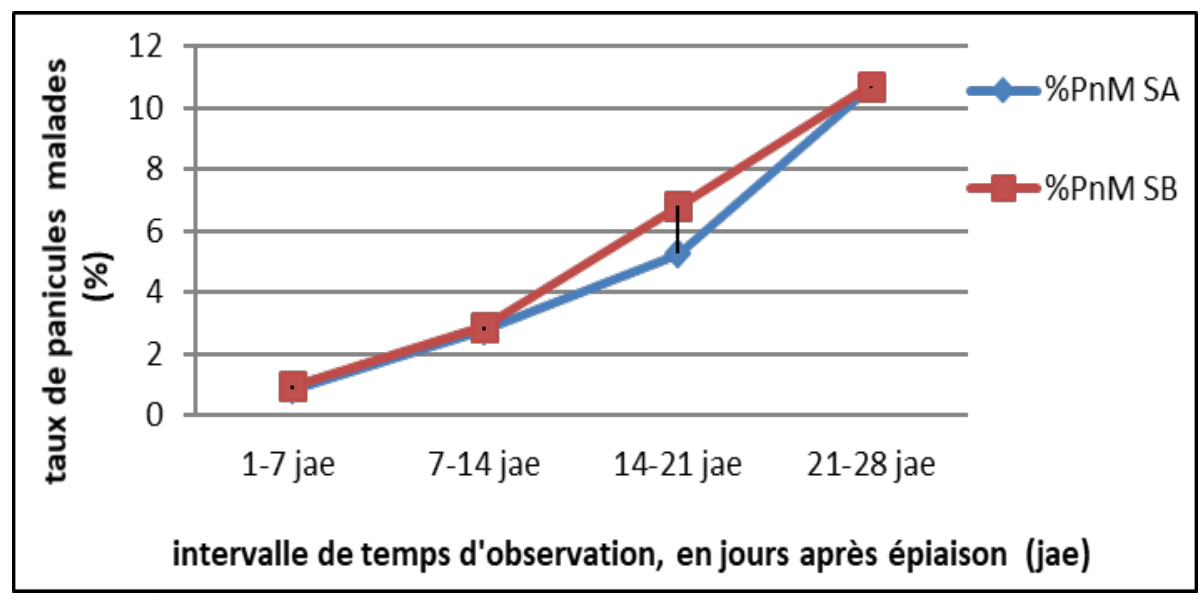

Figure 2 : Courbes d'évolution de maladie sur les panicules durant les deux saisons 
Tableau 3. Incidence de pyriculariose paniculaire

\begin{tabular}{|c|c|c|c|c|c|c|c|c|}
\hline \multirow[b]{2}{*}{ Variété } & \multicolumn{5}{|c|}{$\%$ PnM en SA } & \multicolumn{3}{|c|}{$\%$ de progression de maladie } \\
\hline & $7 \mathrm{JAE}$ & 14JAE & 21JAE & $28 \mathrm{JAE}$ & MV & r1 & r2 & r3 \\
\hline Lienge & $0,80 \mathrm{c}$ & $3,24 a$ & $6,71 b$ & $13,77 a$ & 6,1 & 0,35 & 0,49 & 1,01 \\
\hline Lioto & $0,21 \mathrm{e}$ & $2,53 b$ & $3,80 d$ & $11,35 b$ & 4,47 & 0,33 & 0,18 & 1,08 \\
\hline Nerica7 & $0,69 c$ & $2,03 b$ & $3,60 d$ & $8,52 \mathrm{c}$ & 3,7 & 0,19 & 0,23 & 0,70 \\
\hline Liboga & $1,05 b$ & $2,67 a b$ & $4,22 \mathrm{~cd}$ & $6,16 d$ & 3,5 & 0,23 & 0,22 & 0,28 \\
\hline Kitombe & $1,49 a$ & $3,31 a$ & $7,98 a$ & $13,36 a$ & 6,51 & 0,27 & 0,67 & 0,77 \\
\hline $\mathrm{CV}(\%)$ & 8,33 & 10,90 & 7,79 & 10,63 & & & & \\
\hline $\mathrm{S}^{2}$ & 0,87 & 1,12 & 15,44 & 42,45 & & & & \\
\hline \multirow[t]{2}{*}{ LSD $5 \%$} & $0,15^{* *}$ & $0,65^{\star \star *}$ & $0,89^{* * *}$ & $1,50^{\star * *}$ & & & & \\
\hline & \multicolumn{3}{|c|}{$\%$ PnM en SB } & \multicolumn{4}{|c|}{$\%$ de progression de maladie } & \\
\hline Lienge & $0,85 c$ & $3,26 b$ & $13,41 \mathrm{a}$ & $13,3 a$ & 7,7 & 0,34 & 1,45 & $-0,02$ \\
\hline Lioto & $0,55 \mathrm{e}$ & $2,64 d$ & $3,92 \mathrm{dc}$ & $12,26 a$ & 3,9 & 0,30 & 0,18 & 1,19 \\
\hline Nerica7 & $0,74 d$ & $2,30 \mathrm{e}$ & $3,61 d$ & $8,47 b$ & 3,78 & 0,22 & 0,19 & 0,69 \\
\hline Liboga & $1,18 b$ & $2,79 c$ & $4,46 c$ & $6,42 \mathrm{c}$ & 3,7 & 0,23 & 0,24 & 0,28 \\
\hline Kitombe & $1,35 a$ & $3,41 a$ & $8,5 b$ & $13,01 \mathrm{a}$ & 6,5 & 0,29 & 0,73 & 0,64 \\
\hline $\mathrm{CV}(\%)$ & 5,32 & 0,41 & 5,40 & 5,79 & & & & \\
\hline$S^{2}$ & 0,4 & 0,89 & 70,70 & 37,78 & & & & \\
\hline LSD $5 \%$ & $0,10^{* * *}$ & $0,026^{* * *}$ & $0,62^{* * *}$ & $1,35^{\star * *}$ & & & & \\
\hline
\end{tabular}

Sur la même colonne les valeurs portant les mêmes lettres ne sont pas significativement différentes au seuil de 5\%, $M V=$ moyenne variétale, $J A E=$ jour après épiaison, ${ }^{* * *}=$ différence significative au seuil de $0,1 \%, P n M=$ panicule malade, $S A=$ saison $A, S B=$ saison $B$.

Comportement à la verse: La verse se définie comme une propension plus ou moins grande des variétés de riz à se pencher au sol pendant son plein développement végétatif (Bodelmann, 1976) mais plus particulièrement au remplissage de grains et à la maturité. Dans cette étude la saison n'a pas influencé le comportement de variété à la verse de façon générale, sauf pour la variété Lienge avec moins de $6 \%$ des plants couchés en saison A. En effet, 6 à $25 \%$ des plants de variété Kitombe ont couché durant les deux saisons tandis que les variétés Lioto, Liboga et Nerica7 ont été moins affectées avec moins de $2 \%$ des plantes couchées (tableau 4).La constitution anatomique des tiges (diamètre, épaisseur des faisceaux fibrovasculaire, l'importance de lacune centrale et ainsi que le format de la feuille) est à la base de la verse du point de vu variétal (Bodelmann,1976) et les conditions climatiques particulièrement les pluies et les vents du point de vue environnemental. Cependant, nous avons constaté que la taille des plants combinée au caractère pubescent des feuilles et des grains qui ont pu charger de l'eau après la pluie ou la rosée a beaucoup contribué à ces résultats de Kitombe. L'environnement aurait moins agit car les essais ont été installés dans les bandes herbeuses qui ont certainement joué le rôle de rideau.

Tableau 4. Valeurs moyennes de l'évolution de l'aire de l'épidémie et de la verse.

\begin{tabular}{clcccccc}
\hline \multirow{2}{*}{ Code essai } & Variété & \multicolumn{2}{c}{ AUDPC Foliaire } & \multicolumn{2}{c}{ AUDPC Paniculaire } & \multicolumn{2}{c}{ Verse (1-9) a } \\
\cline { 2 - 7 } v1 & SA & SB & SA & SB & SA & SB \\
v2 & Lioto & 48,60 & 66,6 & 51,92 & 49,8 & 5 & 1 \\
v3 & Nerica7 & 76,76 & 78,3 & 44,56 & 46,84 & 1 & 1 \\
v4 & Liboga & 95,91 & 82,8 & 31,36 & 30,92 & 1 & 1 \\
v5 & Kitombe & 109,16 & 77,85 & 20,44 & 20,96 & 1 & 1 \\
\hline
\end{tabular}

a Echelle visuelle d'évaluation de la verse du centre du riz pour l'Afrique, ADRAO (ADRAO, 2009) 
Résistance à l'égrainage : La résistance à l'égrainage a été testée au champ à différents degré de maturité et est indiquée par les figures 3 et 4 . Ces figures montrent que les variétés Lienge et Nerica7 sont des variétés très sensibles à l'égrenage $(p<0,001)$ et cette sensibilité évolue proportionnellement avec le degré de maturité. L'égrainage est un caractère essentiellement variétal dû à la conformation et surtout à la texture des tissus formant la capicuule, support qui relie le pédicelle à l'épillet. Les variétés possédant une facette oblique (cas des variétés Liboga, Lioto et Kitombe) sont, selon Bodelmann (1976) en générale peu égrenables tandis que les variétés ayant une facette horizontale (cas de variétés Lienge et Nerica7) s'égrènent facilement.

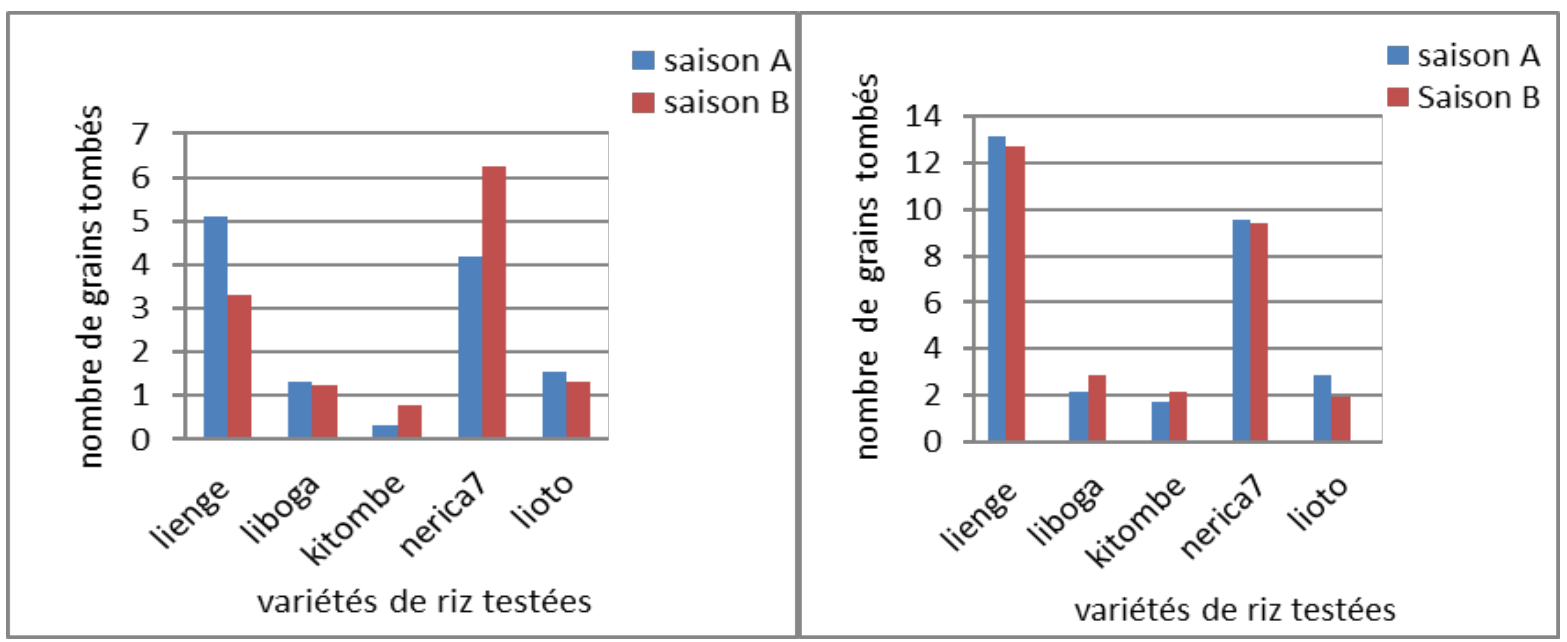

Figure 3: Histogrammes de distribution des génotypes en fonction de leur égrenabilité à maturité $80 \%(n=40)$

Figure 4: Histogrammes de distribution des génotypes en fonction de leur égrenabilité à maturité $100 \% \quad(n=40)$

Tableau 5: Analyse de variance (ANOVA) du paramètre " résistance à l'égrenage »

\begin{tabular}{lcccc}
\hline & \multicolumn{2}{c}{ Maturité $\mathbf{8 0} \%$} & \multicolumn{2}{c}{ Maturité $\mathbf{1 0 0 \%}$} \\
\cline { 2 - 5 } Paramètres statistiques & SA & SB & SA & SB \\
\hline Moyenne Générale & 1,90 & 2,23 & 4,35 & 4,42 \\
S $^{2}$ & 9,15 & 15,25 & 53,28 & 60,14 \\
C.V (\%) & 22,00 & 24,21 & 19,31 & 19,16 \\
LSD 5\% & $0,71^{* * *}$ & $1,17^{* * *}$ & $1,83^{\star * *}$ & $1,91^{* * *}$ \\
\hline
\end{tabular}

${ }^{* \star *}=$ différence significative au seuil de $0,1 \%$,

\section{REMERCIEMENTS}

Cette recherche a été supportée par le fondation internationale pour la science (IFS) Stockholm, Suède à

\section{CONCLUSION ET APPLICATION DES RESULTATS}

La résistance du riz à la pyriculariose se classe à deux types, qualitative (complète ou verticale) et quantitative (partielle ou horizontale). Dans cette étude, l'objectif était de connaitre le comportement de cinq variétés de riz pluvial à la pyriculariose et à la verse dans les conditions naturelles à Kisangani. II a été constaté des comportements diversifiés et des écarts élevés entre les notes minimales et maximales obtenues par les travers la bourse accordée à Monsieur Bienvenu Liboga

différentes variétés. Certaines variétés ont manifesté un comportement d'hypersensibilité, ce qui a permis de réduire la progression de la maladie, avec une valeur faible de l'Area Under the disease progress curve (AUDPC) entrainant ainsi un faible taux de surface foliaire malade. C'est le cas de variété Lienge sur les feuilles en saison $A$ et $B$ ainsi que sur les panicules en saison B. La pyriculariose paniclulaire est celle qui 
affecte directement la production, car elle entraine la non remplissage de grains, la mauvaise sortie de panicule, la décoloration de grains, diminution de poids de mille grains. .Les valeurs moyennes d'incidence de pyriculariose paniculaire obtenues par les variétés Liboga et Nerica7 durant les deux essais ne dépassent pas $4 \%$, en conséquence Liboga et Nerica7 peuvent être considérées comme des variétés ayant un bon niveau de résistance quantitative qui s'exprime comme une résistance de type horizontal, caractérisée par une résistance stable dans différentes conditions. Les

\section{REFERENCES BIBLIOGRAPHIQUES}

Ashkani S., Rafii M.Y., Shabanimofrad M., Ghasemzadeh A., Ravanfar S.A., Latif M.A.2016 Molecular progress on the mapping and cloning of functional genes for blast disease in rice (Oryza sativa L.): current status and future considerations. Critical Reviews in Biotechnology 36(2)353-367, DOI:10.3109/07388551.2014.961403.

Babiker A.G., Samejima H., Mustafa A., Sugimoto Y.2016. Identification of Striga hermonthicaResistant Upland Rice Varieties in Sudan and Their Resistance Phenotypes Front. Plant Sci. 7:634. DOI:10.3389/fpls.2016.00634.

Bangata B.M., Ngbolua K.N., Ekustu E. et Kalonji A.M.2013. Comportement de quelques lignées de riz Nerica en culture de bas-fond dans la région de Kinshasa, République Démocratique du Congo. Int. J. Biol. Chem. Sci. 7(1): 25-32. DOI: dx.doi.org/10.4314/ijbcs.v7i1i.3.

Bodelmann J.P.1976. Riziculture pratique I, riz irrigué. Presses Universitaires de France.

Bouet A., Amancho N. A.2010. Sélection de deux nouvelles variétés de riz irrigue (Oryza sativa L.) a un haut niveau de résistance au virus de la panachure jaune (RYMV) en côte d'ivoire. Agronomie Africaine 22 (3):285-293.

Centre du riz pour l'Afrique (ADRAO) ,2009. Sélection variétale participative du riz: Manuel du technicien. Cotonou, Benin: Centre du riz pour l'Afrique (ADRAO) 126pp.

Gnago A.J., Kouadio K.T., Tia V.E., Kodro A.P., Goulivas A.V.2017. Évaluation de deux variétés de riz (CK73 et CK90) à la Toxicité Ferreuse et à quelques contraintes biotiques à Yamoussoukro (Côte d'lvoire) J. Appl. Biosci. 112:11035-11044 DOI:doi.org/10.4314/jab.v112i1.8. valeurs minimales de la verse, évaluée avec l'échelle de I'ADRAO a permis de classer les variétés Lioto, Liboga et Nerica7 comme résistantes et Kitombe moyennement sensible. Une attention particulière doit être accordée à la maturité de la variété Lienge et Nerica7 qui font tomber beaucoup de grains à ce stade. Pour cela, la récolte de ces variétés ne doit pas être trop tardive. Cette étude a offert des informations utiles pour la production végétale dans l'agriculture biologique en sélectionnant les variétés résistantes.

International Rice Research Institute (IRRI),2002. Standard evaluation system for rice (SES). Manila, Philippines, 56pp.

Islam M. M., Kayesh E., Zaman E., Urmi T.A., Haque M.M.2018. Evaluation of Rice (Oryza sativa L.) Genotypes for Drought Tolerance at Germination and Early Seedling Stage. The Agriculturists 16(1):44-54.

Kasongo K.M., Walangululu M.J., Bantodisa K.M., Likoko, B., Mbuya K.2003. Etude du comportement et des performances de huit lignées hybrides de riz pluvial à cycle moyen sélectionnées à Yangambi. Tropicultura, 21(3):112-116

Kim H.J., Han S-S. Kwon S-J. Choi H-C., Hwang H-G. and Ahn S-N. 2003. Genetic Diversity of Blast resistance and selected Korean rice cultivars based on simple sequence repeats. Koean J.Breed.35 (3):170-178.

Leila A G.,Sitarama P A.,Pedro Arraes PA.2004.RAPD marker linked to a gene conferring resistance to race $\mathrm{IB}-9$ of Pyricularia grisea in a somaclone of the rice cultivar. Plant Cell, Tissue and Organ Culture 78:151-158.

Lepoive P.2003. Phytopathologie, Presses agronomiques de Gembloux, édition de boeck.427pp

Louvel D.1981.Héritabilité de la résistance au champ de variétés de riz pluvial vis-à-vis de la Pyricularia oryzae en Côte d'lvoire. In: comptes rendus du symposium sur la résistance du riz à la pyriculariose, Montpellier, France, du 18-21 mars, p.319-329, Montpellier, IRAT.

Momolu E.P., Katuromunda S., Lam J.2016. Effect of Soil Moisture Stress Duration on the Growth Characteristics and Yield of Rice Cultivars. J. 
$\begin{array}{llll}\text { Agri. and Env. Sci.5 (2): 66-76. } & \end{array}$ DOI:10.15640/jaes.v5n2a9.

Naqvi N.I.,Bonman M.,Mackill D.J.,Nelson R.J.,Chattoo BB.1995.Indentification of RAPD markers linked to a major blast resistance gene in rice. Molecular breeding, 1:341-348.

Notteghen JL.1981.Analyse de résultats d'inoculation de 67 variétés de riz par 15 souches de Pyricularia oryzae. In: comptes rendus du symposium sur la résistance du riz à la pyriculariose, Montpellier, France, du 18-21 mars, p.319-329, Montpellier, IRAT.

Ouédraogo M., Dakouo D.2017.Evaluation de l'adoption des variétés de riz Nerica dans I'Ouest de Burkina-Faso, AfJARE, vol.12, No1.

Ouédraogo M.2008.Incidence de l'helminthosporiose du riz au Burkina Faso et caractérisation des populations de l'agent pathogène [Bipolaris oryzae (Breda de Haan) Shoemaker].Thèse de doctorat, Université de Thiès, Sénégal.

Parlevliet J.E.1983.Can horizontal resistance be recognized in the presence of vertical resistance in plant exposed to un mixture race? Plant disease 73:p, 379

Samota M.K., Sasi M., Singh A. 2017.Impact of Seed Priming on Proline Content and Antioxidant Enzymes to Mitigate Drought Stress in Rice Genotype Int.J.Curr.Microbio.App.Sci 6(5):2459-2466.

DOI:doi.org/10.20546/ijcmas.2017.605.275

Shaner G., Finney R.E., 1977.The effect of nitrogen fertilization on the expression of slow mildewing resistance in Knox wheat. Phytopathology $67: 1051-1056$.

Van Wambeke A. et Libens R.1957. Carte des sols et de la végétation du Congo-Belge et $\mathrm{du}$ Rwanda-Urundi. Bruxelles; 47p

Yu Z.H.,Mackill D.J.,Bonman J.M., Tanksley SD.1991.Tagging genes for resistance in rice via linkage to RFLP markers. Theor.Appl.Genet.81:471- 476. 